\title{
The Explicit Expression of the Drazin Inverse and Its Application
}

\author{
Xiaoji Liu, ${ }^{1}$ Shaowu Huang, ${ }^{1}$ Liang Xu, ${ }^{1}$ and Yaoming $\mathrm{Yu}^{2}$ \\ ${ }^{1}$ School of Science, Guangxi University for Nationalities, Nanning 530006, China \\ ${ }^{2}$ School of Mathematical Sciences, Monash University, VIC 3800, Australia \\ Correspondence should be addressed to Xiaoji Liu; xiaojiliu72@126.com
}

Received 18 July 2013; Accepted 6 August 2013

Academic Editor: Renat Zhdanov

Copyright (c) 2013 Xiaoji Liu et al. This is an open access article distributed under the Creative Commons Attribution License, which permits unrestricted use, distribution, and reproduction in any medium, provided the original work is properly cited.

We give explicit expressions of $(P \pm Q)_{d}$ of two matrices $P$ and $Q$, in terms of $P, Q, P_{d}$, and $Q_{d},(P \pm I)_{d}$, under the condition that $P Q=P$, and apply the result to finding an explicit representation for the Drazin inverse of some $2 \times 2$ block matrix.

\section{Introduction}

In recent years, the representations and perturbation analysis of the Drazin inverse for matrices or operators have been investigated (see [1-6]). In [7], the author presented the presentations of the Drazin inverse of sum and product of two operators over Banach spaces on the condition of the commutativity up to a factor. And, in [8], the same author discussed explicit representations of Drazin inverses of sums and differences of two idempotents over Hilbert spaces.

These investigations motivate us to deal with an explicit expression of the Drazin inverse of differences and sums of two matrices. The paper is organized as follows. In this section, we will introduce some notions and lemmas. In Section 2, we will present these explicit expressions of differences and sums of two matrices $P$ and $Q$ under the condition $P Q=P$. In Section 3, we will deduce an explicit representation for the Drazin inverse of the $2 \times 2$ block matrix $\left[\begin{array}{ll}A & B \\ C & D\end{array}\right]$ with $A=B C$ and $B=B D$ in terms of its subblocks and their Drazin inverses and $(A+I)_{d}$. In Section 4, we will present a numerical example to demonstrate the main result in Section 2.

Throughout this paper the symbol $\mathbb{C}^{m \times n}$ stands for the set of $m \times n$ complex matrices, and $I \in \mathbb{C}^{n \times n}$ stands for the unit matrix. Let $A \in \mathbb{C}^{n \times n}$; the Drazin inverse, denoted by $A_{d}$, of matrix $A$ is defined as the unique matrix satisfying

$$
A^{k+1} A_{d}=A^{k}, \quad A_{d} A A_{d}=A_{d}, \quad A A_{d}=A_{d} A
$$

where $k=\operatorname{Ind}(A)$ is the index of $A$. In particular, if $\operatorname{Ind}(A)=$ 1 , then $A_{d}$ is called the group inverse, denoted by $A_{g}$, of $A$. Apparently, if $A$ is nonsingular, then $\operatorname{Ind}(A)=0$; otherwise, $\operatorname{Ind}(A) \geq 1$, especially $\operatorname{Ind}(0)=1$. If $A$ is nilpotent, then $A_{d}=$ 0 . If $X$ is nonsingular and $B=X A X^{-1}$, then $B_{d}=X A_{d} X^{-1}$ (see $[6,9,10]$ ). For convenience, we write $A^{\pi}=I-A A_{d}$ and use the convention $\sum_{i=n}^{m}=0$, if $m<n$.

Before we start the discussion, we need some preparations.

Lemma 1 (see [11, Theorem 3.2]). Let $M=\left[\begin{array}{cc}A & B \\ 0 & D\end{array}\right]$ with $t=$ $\operatorname{Ind}(A)$ and $l=\operatorname{Ind}(D)$. Then,

$$
M_{d}=\left[\begin{array}{cc}
A_{d} & S \\
0 & D_{d}
\end{array}\right]
$$

where

$$
S=A^{\pi} \sum_{n=0}^{t-1} A^{n} B D_{d}^{n+2}+\sum_{n=0}^{l-1} A_{d}^{n+2} B D^{n} D^{\pi}-A_{d} B D_{d} .
$$

Lemma 2. Let $P, Q \in \mathbb{C}^{m \times m}$ with $\operatorname{Ind}(Q)=s$. If $P Q=P$, then

$$
\begin{gathered}
P Q_{d}=P, \\
\left(Q_{d} Q P\right)^{k}=Q_{d} Q P^{k}, \quad\left(Q P^{\pi}\right)^{k}=Q^{k} P^{\pi}, \\
\left(Q_{d} P^{\pi}\right)^{k}=Q_{d}^{k} P^{\pi}, \quad k \geq 1 .
\end{gathered}
$$


Proof. Since $P Q=P$ and $\operatorname{Ind}(Q)=s$,

$$
P=P Q^{s}=P Q^{s+1} Q_{d}=P Q_{d}
$$

and then $P Q Q_{d}=P$. So from this, by induction, it follows that $\left(Q_{d} Q P\right)^{k}=Q_{d} Q P^{k}$ for $k \geq 1$.

Now, we will show inductively that $\left(Q P^{\pi}\right)^{k}=Q^{k} P^{\pi}$. It holds for $k=1$. Assume it holds for $k=n$; that is, $\left(Q P^{\pi}\right)^{n}=$ $Q^{n} P^{\pi}$. Then,

$$
\left(Q P^{\pi}\right)^{n+1}=\left(Q P^{\pi}\right)\left(Q P^{\pi}\right)^{n}=Q\left(I-P_{d} P\right) Q^{n} P^{\pi}=Q^{n+1} P^{\pi} .
$$

So it holds for any $k \geq 1$.

From $P Q_{d}=P$, we can similarly show that $\left(Q_{d} P^{\pi}\right)^{k}=$ $Q_{d}^{k} P^{\pi}$.

Lemma 3. Let $P$ be nilpotent of index $t>1$ and $S=$ $\sum_{i=0}^{t-1} a_{i}^{[1]} P^{i}$. If $a_{i}^{[1]}=1$, then

$$
S^{n}=\sum_{i=0}^{t-1} a_{i}^{[n]} P^{i}, \quad n \geq 2,
$$

where $a_{i}^{[n]}=\sum_{u=0}^{i} a_{u}^{[n-1]}, i=0, \ldots, t-1$.

Proof. Since $P^{t}=0$, we can easily write $S^{n}$ as

$$
S^{n}=\sum_{i=0}^{t-1} a_{i}^{[n]} P^{i}, \quad n \geq 2 .
$$

We will prove the relationship

$$
a_{i}^{[n]}=\sum_{h=0}^{i} a_{h}^{[n-1]}, \quad i=0, \ldots, t-1
$$

by induction on $n$. Obviously, (9) holds for $n=2$. Assume inductively that (9) holds for $n=k$.

Since $a_{i}^{[1]}=1$ and $P^{t}=0$,

$$
\begin{aligned}
S^{k+1} & =S^{k} S=\sum_{i=0}^{t-1} a_{i}^{[k]} P^{i} \sum_{j=0}^{t-1} P^{j} \\
& =\sum_{h=0}^{t-1} \sum_{i+j=h} a_{i}^{[k]} P^{i+j}=\sum_{h=0}^{t-1} \sum_{i=0}^{h} a_{i}^{[k]} P^{h} .
\end{aligned}
$$

On the other hand,

$$
S^{k+1}=\sum_{i=0}^{t-1} a_{i}^{[k+1]} P^{i} .
$$

So, (9) holds for $n=k+1$. Hence, (9) holds for $n \geq 2$.

Lemma 4 (see [9, Lemma 7.7.2]). Let

$$
N=\left[\begin{array}{cc}
A & B \\
0 & 0
\end{array}\right] \quad \text { or } N=\left[\begin{array}{ll}
0 & 0 \\
B & A
\end{array}\right] \text {. }
$$

Then, $\operatorname{Ind}(A) \leq \operatorname{Ind}(N) \leq \operatorname{Ind}(A)+1$.

\section{The Drazin Inverse of Differences and Sums of Two Matrices}

In this section, we will investigate how to express $P \pm Q$ as a function of $P, Q, P_{d}$, and $Q_{d},(P \pm I)_{d}$, under the condition $P Q=P$. We begin with the following theorem, in which $P$ is assumed to be nilpotent.

Theorem 5. Let $P, Q \in \mathbb{C}^{n \times n}$ with $\operatorname{Ind}(Q)=$ s. If $P Q=P$ and $P$ is nilpotent of index $t$, then

$$
\begin{aligned}
(P-Q)_{d}= & \sum_{n=0}^{s-1} \sum_{i=0}^{t-2}\left(a_{i}^{[n+2]} I-a_{i}^{[n+1]} Q_{d}\right) Q^{n} P^{i+1} \\
& -Q_{d} Q^{s} \sum_{i=0}^{t-2} a_{i}^{[s+1]} P^{i+1}-Q_{d}
\end{aligned}
$$

where $a_{i}^{[n]}=\sum_{k=0}^{i} a_{k}^{[n-1]}$ and $a_{i}^{[1]}=1, i=0, \ldots, t-1$.

Proof. If $t=1$, then, from the convention in Section 1, (13) clearly holds. Now assume $t>1$. From $s=\operatorname{Ind}(Q)$, there exists a nonsingular matrix $W_{1}$ such that

$$
\begin{aligned}
& Q=W_{1}\left[\begin{array}{cc}
Q_{1} & 0 \\
0 & Q_{2}
\end{array}\right] W_{1}^{-1}, \\
& Q_{d}=W_{1}\left[\begin{array}{cc}
Q_{1}^{-1} & 0 \\
0 & 0
\end{array}\right] W_{1}^{-1},
\end{aligned}
$$

where $Q_{1}$ is nonsingular and $Q_{2}$ is nilpotent of index $s$. Partitioning $W_{1}^{-1} P W_{1}$ conformably with $W_{1}^{-1} Q W_{1}$, we have

$$
P=W_{1}\left[\begin{array}{ll}
P_{1} & P_{4} \\
P_{3} & P_{2}
\end{array}\right] W_{1}^{-1}
$$

Since $P Q=P, P Q_{d}=P$ by Lemma 2 , and then $P_{2}=0, P_{4}=0$, and $P_{i} Q_{1}=P_{i}, i=1,3$. Let $t$ be the index of nilpotent matrix $P$. Then,

$$
P^{t}=W_{1}\left[\begin{array}{cc}
P_{1}^{t} & 0 \\
P_{3} P_{1}^{t-1} & 0
\end{array}\right] W_{1}^{-1}=0,
$$

and therefore $P_{1}$ is also nilpotent, and $\left(P_{1}-I\right)^{-1}=-\sum_{i=0}^{t-1} P_{1}^{i}$. Thus,

$$
\begin{aligned}
P-Q & =W_{1}\left[\begin{array}{cc}
P_{1}-Q_{1} & 0 \\
P_{3} & -Q_{2}
\end{array}\right] W_{1}^{-1} \\
& =W_{1}\left[\begin{array}{cc}
\left(P_{1}-I\right) Q_{1} & 0 \\
P_{3} & -Q_{2}
\end{array}\right] W_{1}^{-1}
\end{aligned}
$$

By Lemma 1, we have

$$
\begin{aligned}
(P-Q)_{d} & =W_{1}\left[\begin{array}{cc}
Q_{1}^{-1}\left(P_{1}-I\right)^{-1} & 0 \\
X & 0
\end{array}\right] W_{1}^{-1} \\
& =W_{1}\left[\begin{array}{cc}
-Q_{1}^{-1} \sum_{i=0}^{t-1} P_{1}^{i} & 0 \\
X & 0
\end{array}\right] W_{1}^{-1},
\end{aligned}
$$


where

$$
X=\sum_{n=0}^{s-1}(-1)^{n} Q_{2}^{n} P_{3}\left[\left(P_{1}-I\right) Q_{1}\right]^{-(n+2)} .
$$

By Lemma 3,

$$
\begin{aligned}
(-1)^{n} P_{3}\left[\left(P_{1}-I\right) Q_{1}\right]^{-(n+2)} & =P_{3}\left(Q_{1}^{-1} \sum_{i=0}^{t-1} P_{1}^{i}\right)^{n+2} \\
& =P_{3}\left(\sum_{i=0}^{t-1} P_{1}^{i}\right)^{n+2} \\
& =P_{3} \sum_{i=0}^{t-1} a_{i}^{[n+2]} P_{1}^{i} \\
& =\sum_{i=0}^{t-2} a_{i}^{[n+2]} P_{3} P_{1}^{i}
\end{aligned}
$$

Thus,

$$
\begin{aligned}
W_{1}\left[\begin{array}{ll}
0 & 0 \\
X & 0
\end{array}\right] W_{1}^{-1} & =\sum_{n=0}^{s-1} \sum_{i=0}^{t-2} W_{1}\left[\begin{array}{cc}
0 & 0 \\
a_{i}^{[n+2]} Q_{2}^{n} P_{3} P_{1}^{i} & 0
\end{array}\right] W_{1}^{-1} \\
& =\sum_{n=0}^{s-1} \sum_{i=0}^{t-2} a_{i}^{[n+2]} Q^{\pi} Q^{n} P^{i+1} .
\end{aligned}
$$

So, by (18),

$$
\begin{aligned}
(P-Q)_{d}= & -\sum_{i=0}^{t-1} Q_{d} P^{i}+Q^{s} \sum_{n=0}^{s-1} \sum_{i=0}^{t-2} a_{i}^{[n+2]} Q^{n} P^{i+1} \\
= & -\sum_{i=0}^{t-2} Q_{d} P^{i+1}-Q_{d} \\
& +\sum_{n=0}^{s-1} \sum_{i=0}^{t-2} a_{i}^{[n+2]} Q^{n} P^{i+1} \\
& -Q_{d} \sum_{n=1}^{s} \sum_{i=0}^{t-2} a_{i}^{[n+1]} Q^{n} P^{i+1} \\
= & -Q_{d}+\sum_{n=0}^{s-1} \sum_{i=0}^{t-2} a_{i}^{[n+2]} Q^{n} P^{i+1} \\
& -Q_{d} \sum_{n=0}^{s} \sum_{i=0}^{t-2} a_{i}^{[n+1]} Q^{n} P^{i+1} \\
= & \sum_{n=0}^{s-1} \sum_{i=0}^{t-2}\left(a_{i}^{[n+2]} I-a_{i}^{[n+1]} Q_{d}\right) Q^{n} P^{i+1} \\
& -Q_{d} Q^{s} \sum_{i=0}^{t-2} a_{i}^{[s+1]} P^{i+1}-Q_{d} .
\end{aligned}
$$

If $P Q=P$, then $(-P) Q=-P$. So, it immediately follows from the above theorem.
Corollary 6. Let $P, Q \in \mathbb{C}^{n \times n}$ with $\operatorname{Ind}(Q)=$ s. If $P Q=P$ and $P$ is nilpotent of index $k$, then

$$
(P+Q)_{d}=\sum_{n=0}^{s-1} \sum_{i=0}^{t-2}(-1)^{i}\left(a_{i}^{[n+2]} I-a_{i}^{[n+1]} Q_{d}\right) Q^{n} P^{i+1}
$$$$
-Q_{d} Q^{s} \sum_{i=0}^{t-2}(-1)^{i} a_{i}^{[s+1]} P^{i+1}+Q_{d},
$$

where $a_{i}^{[n]}=\sum_{k=0}^{i} a_{k}^{[n-1]}, a_{i}^{[1]}=1, i=0, \ldots, t-1$.

If the nilpotency of $P$ is taken out in Theorem 5, then we can obtain our main result, a more general result.

Theorem 7. Let $P, Q \in \mathbb{C}^{n \times n}$ with $\operatorname{Ind}\left(Q P^{\pi}\right)=s$, $\operatorname{Ind}(P)=t$, $\operatorname{Ind}\left[(P-I) P P_{d}\right]=l$, and $\operatorname{Ind}\left[(P-Q) P^{\pi}\right]=k$. If $P Q=P$, then

$$
\begin{aligned}
(P-Q)_{d}= & \sum_{n=0}^{s-1} \sum_{i=0}^{t-2}\left(a_{i}^{[n+2]} I-a_{i}^{[n+1]} Q_{d}\right) Q^{n} P^{i+1} P^{\pi}-Q_{d} P^{\pi} \\
& -\sum_{n=0}^{k-1}(-1)^{n} Q^{n} Q^{\pi}(Q-I) P P_{d}(P-I)_{d}^{n+2} \\
& +\left(Q^{\pi}+Q_{d}\right) P P_{d}(P-I)_{d} \\
& +\sum_{n=0}^{l-1}(-1)^{n+1} Q_{d}^{n}\left(Q_{d}-Q_{d}^{2}\right) P P_{d}(P-I)^{n}(P-I)^{\pi} \\
& -Q_{d} Q^{s} \sum_{i=0}^{t-2} a_{i}^{[s+1]} P^{\pi} P^{i+1},
\end{aligned}
$$

where $a_{i}^{[n]}=\sum_{k=0}^{i} a_{k}^{[n-1]}, a_{i}^{[1]}=1, i=0, \ldots, t-1$.

Proof. There exists a nonsingular matrix $W_{1}$ such that

$$
P=W_{1}\left[\begin{array}{cc}
P_{1} & 0 \\
0 & P_{2}
\end{array}\right] W_{1}^{-1}, \quad Q=W_{1}\left[\begin{array}{ll}
Q_{1} & Q_{3} \\
Q_{4} & Q_{2}
\end{array}\right] W_{1}^{-1},
$$

where $P_{1}$ is nonsingular and $P_{2}$ is nilpotent of index $t$. From $P Q=P$, we get

$$
Q=W_{1}\left[\begin{array}{cc}
I & 0 \\
Q_{4} & Q_{2}
\end{array}\right] W_{1}^{-1},
$$

where $P_{2} Q_{4}=0$ and $P_{2} Q_{2}=P_{2}$. So

$$
P-Q=W_{1}\left[\begin{array}{cc}
P_{1}-I & 0 \\
-Q_{4} & P_{2}-Q_{2}
\end{array}\right] W_{1}^{-1} .
$$

By Lemma 1, we have

$$
(P-Q)_{d}=W_{1}\left[\begin{array}{cc}
\left(P_{1}-I\right)_{d} & 0 \\
X & \left(P_{2}-Q_{2}\right)_{d}
\end{array}\right] W_{1}^{-1},
$$


where

$$
\begin{aligned}
X= & \sum_{n=0}^{l-1}\left(P_{2}-Q_{2}\right)_{d}^{n+2}\left(-Q_{4}\right)\left(P_{1}-I\right)^{n}\left(P_{1}-I\right)^{\pi} \\
& +\left(P_{2}-Q_{2}\right)^{\pi} \sum_{n=0}^{k-1}\left(P_{2}-Q_{2}\right)^{n}\left(-Q_{4}\right)\left(P_{1}-I\right)_{d}^{n+2} \\
& -\left(P_{2}-Q_{2}\right)_{d}\left(-Q_{4}\right)\left(P_{1}-I\right)_{d},
\end{aligned}
$$

and $k=\operatorname{Ind}\left(P_{2}-Q_{2}\right)=\operatorname{Ind}\left[(P-Q) P^{\pi}\right]$ and $l=\operatorname{Ind}\left(P_{1}-I\right)=$ $\operatorname{Ind}\left[(P-I) P P_{d}\right]$.

Since $P_{2} Q_{4}=0$ and $P_{2} Q_{2}=P_{2}$, by Theorem 5 and Lemma 2 ,

$$
\begin{aligned}
\left(P_{2}-Q_{2}\right)_{d}\left(Q_{2}\right)_{d}^{j} Q_{4}= & -\sum_{n=0}^{t-1}\left(Q_{2}\right)_{d} P_{2}^{n}\left(Q_{2}\right)_{d}^{j} Q_{4} \\
& +\sum_{n=0}^{s-1} Q_{2}^{\pi} Q_{2}^{n} P_{2}\left(\sum_{i=0}^{t-2} P_{2}^{i}\right)^{n+2}\left(Q_{2}\right)_{d}^{j} Q_{4} \\
= & -\left(Q_{2}\right)_{d}^{j+1} Q_{4}, \quad j \geq 0, \\
\left(P_{2}-Q_{2}\right)^{\pi} Q_{4}= & Q_{4}+\left(P_{2}-Q_{2}\right)\left(Q_{2}\right)_{d} Q_{4} \\
= & Q_{4}-Q_{2}\left(Q_{2}\right)_{d} Q_{4} \\
& =Q_{2}^{\pi} Q_{4}, \\
& P_{2} Q_{2}^{\pi}=0,
\end{aligned}
$$

and then

$$
\begin{gathered}
\left(P_{2}-Q_{2}\right)^{\pi} \sum_{n=0}^{k-1}\left(P_{2}-Q_{2}\right)^{n}\left(-Q_{4}\right) \\
=-\sum_{n=0}^{k-1}\left(P_{2}-Q_{2}\right)^{n} Q_{2}^{\pi} Q_{4} \\
=-\sum_{n=0}^{k-1}\left(-Q_{2}\right)^{n} Q_{2}^{\pi} Q_{4} .
\end{gathered}
$$

Thus,

$$
\begin{aligned}
X= & \sum_{n=0}^{l-1}(-1)^{n+1}\left(Q_{2}\right)_{d}^{n+2} Q_{4}\left(P_{1}-I\right)^{n}\left(P_{1}-I\right)^{\pi} \\
& -\sum_{n=0}^{k-1}(-1)^{n} Q_{2}^{n} Q_{2}^{\pi} Q_{4}\left(P_{1}-I\right)_{d}^{n+2} \\
& -\left(Q_{2}\right)_{d} Q_{4}\left(P_{1}-I\right)_{d} .
\end{aligned}
$$

Since

$$
P-I=W_{1}\left[\begin{array}{cc}
P_{1}-I & 0 \\
0 & P_{2}-I
\end{array}\right] W_{1}^{-1},
$$

we have that, for $n \geq 0$,

$$
\begin{aligned}
P P_{d}(P-I)_{d}^{n} & =W_{1}\left[\begin{array}{cc}
\left(P_{1}-I\right)_{d}^{n} & 0 \\
0 & 0
\end{array}\right] W_{1}^{-1}, \\
(P-I)^{n} P P_{d}(P-I)^{\pi} & =W_{1}\left[\begin{array}{cc}
\left(P_{1}-I\right)^{n}\left(P_{1}-I\right)^{\pi} & 0 \\
0 & 0
\end{array}\right] W_{1}^{-1} .
\end{aligned}
$$

Obviously,

$$
\begin{gathered}
\left(\mathrm{Q} P^{\pi}\right)_{d}=W_{1}\left[\begin{array}{cc}
0 & 0 \\
0 & \left(Q_{2}\right)_{d}
\end{array}\right] W_{1}^{-1}=Q_{d} P^{\pi}, \\
(Q-I) P P_{d}=W_{1}\left[\begin{array}{cc}
0 & 0 \\
Q_{4} & 0
\end{array}\right] W_{1}^{-1}, \\
Y^{n} P^{\pi}=W_{1}\left[\begin{array}{cc}
0 & 0 \\
0 & Y_{2}^{n}
\end{array}\right] W_{1}^{-1}, \quad n \geq 0,
\end{gathered}
$$

where the symbol $Y$ denotes $Q$ or $P$. Also,

$$
P^{\pi}\left(Q P^{\pi}\right)^{\pi}=P^{\pi}-P^{\pi} Q P^{\pi} Q_{d} P^{\pi}=P^{\pi}-Q Q_{d} P^{\pi}=Q^{\pi} P^{\pi} .
$$

Note that $P^{\pi}(Q-I)=(Q-I)$. Then, by (32),

$$
\begin{aligned}
W_{1}\left[\begin{array}{ll}
0 & 0 \\
X & 0
\end{array}\right] W_{1}^{-1} \\
=\sum_{n=0}^{l-1}(-1)^{n+1} Q_{d}^{n+2} P^{\pi}(Q-I) P P_{d}(P-I)^{n} P P_{d}(P-I)^{\pi} \\
\quad-\sum_{n=0}^{k-1}(-1)^{n} Q^{n} P^{\pi}\left(Q P^{\pi}\right)^{\pi}(Q-I) P P_{d}(P-I)_{d}^{n+2} \\
\quad-Q_{d} P^{\pi}(Q-I) P P_{d}(P-I)_{d} \\
=\sum_{n=0}^{l-1}(-1)^{n+1} Q_{d}^{n+2}(Q-I) P P_{d}(P-I)^{n}(P-I)^{\pi} \\
\quad-\sum_{n=0}^{k-1}(-1)^{n} Q^{n} Q^{\pi}(Q-I) P P_{d}(P-I)_{d}^{n+2} \\
\quad-Q_{d}(Q-I) P P_{d}(P-I)_{d} .
\end{aligned}
$$

Note that $P^{\pi} Q^{n} P^{\pi}=Q^{n} P^{\pi}$. So, by Theorem 5,

$$
\begin{aligned}
& W_{1}\left[\begin{array}{cc}
0 & 0 \\
0 & \left(P_{2}-Q_{2}\right)_{d}
\end{array}\right] W_{1}^{-1} \\
&=\sum_{n=0}^{s-1} \sum_{i=0}^{t-2}\left(a_{i}^{[n+2]} I-a_{i}^{[n+1]} Q_{d} P^{\pi}\right) Q^{n} P^{\pi} P^{i+1} P^{\pi} \\
& \quad-Q_{d} P^{\pi}-Q_{d} P^{\pi} Q^{s} P^{\pi} \sum_{i=0}^{t-2} a_{i}^{[s+1]} P^{i+1} P^{\pi} \\
&=\sum_{n=0}^{s-1} \sum_{i=0}^{t-2}\left(a_{i}^{[n+2]} I-a_{i}^{[n+1]} Q_{d}\right) Q^{n} P^{i+1} P^{\pi} \\
&-Q_{d} P^{\pi}-Q_{d} Q^{s} \sum_{i=0}^{t-2} a_{i}^{[s+1]} P^{\pi} P^{i+1}
\end{aligned}
$$

Hence, putting (34), (37), and (38) into (28) yields (24). 
Corollary 8. Let $P, Q \in \mathbb{C}^{n \times n}$ with $\operatorname{Ind}\left(Q P^{\pi}\right)=s, \operatorname{Ind}(P)=t$, $\operatorname{Ind}\left[(P+I) P P_{d}\right]=l$, and $\operatorname{Ind}\left[(P+Q) P^{\pi}\right]=k$. If $P Q=P$, then

$$
\begin{aligned}
(P & +Q)_{d} \\
= & \sum_{n=0}^{s-1} \sum_{i=0}^{t-2}(-1)^{i}\left(a_{i}^{[n+2]} I-a_{i}^{[n+1]} Q_{d}\right) Q^{n} P^{i+1} P^{\pi}+Q_{d} P^{\pi} \\
& +\sum_{n=0}^{k-1} Q^{n} Q^{\pi}(Q-I) P P_{d}(P+I)_{d}^{n+2} \\
& +\left(Q^{\pi}+Q_{d}\right) P P_{d}(P+I)_{d} \\
& +\sum_{n=0}^{l-1} Q_{d}^{n}\left(Q_{d}-Q_{d}^{2}\right) P P_{d}(P+I)^{n}(P+I)^{\pi} \\
& -Q_{d} Q^{s} \sum_{i=0}^{t-2}(-1)^{i} a_{i}^{[s+1]} P^{\pi} P^{i+1}
\end{aligned}
$$

where $a_{i}^{[n]}=\sum_{k=0}^{i} a_{k}^{[n-1]}, a_{i}^{[1]}=1, i=0, \ldots, t-1$.

\section{The Drazin Inverse of Some $2 \times 2$ Block Matrix}

In this section, we will apply the results in Section 2 to studying the representation for the Drazin inverse of a $2 \times 2$ block matrix, in terms of its subblocks.

Theorem 9. Let $M=\left[\begin{array}{ll}A & B \\ C & D\end{array}\right] \in \mathbb{C}^{n \times n}$ with $\operatorname{Ind}\left[(C B)^{\pi} D\right]=$ $s-1, \operatorname{Ind}(A)=t-1, \operatorname{Ind}\left[(A+I) A A_{d}\right]=l-1$, and Ind $\left[\left[\begin{array}{cc}A^{\pi} & 0 \\ 0 & (C B)^{\pi}\end{array}\right] M\right]=k$. If $A=B C$ and $B=B D$, then

$$
\begin{aligned}
& M_{d} \\
& =\left[\begin{array}{cc}
\left(I-(A+I)_{d}\right)(A+I)_{d} A_{d} & 0 \\
\left(D_{d}+D^{\pi}\right) C(A+I)_{d}^{2} A_{d}+\left(D_{d}^{2}-D_{d}\right) C(A+I)_{d} A_{d} & D_{d}^{2}(C B)^{\pi}
\end{array}\right] M
\end{aligned}
$$

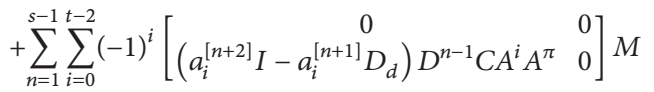

$$
\begin{aligned}
& +\sum_{n=1}^{k-1}\left[\begin{array}{cc}
0 & 0 \\
D^{\pi}\left(D^{n}-D^{n-1}\right) C(A+I)_{d}^{n+2} A_{d} & 0
\end{array}\right] M \\
& +\sum_{n=0}^{l-1}\left[\left(D_{d}^{n+2}-D_{d}^{n+3}\right) C(A+I)^{n}(A+I)^{\pi} A_{d} \quad 0\right] M \\
& +\sum_{i=0}^{t-2}(-1)^{i}\left[\begin{array}{cc}
a_{i}^{[2]} A^{i} A^{\pi} & 0 \\
-\left(a_{i}^{[1]} D_{d}^{2}+a_{i}^{[s+1]} D_{d} D^{s-1}\right) C A^{i} A^{\pi} & 0
\end{array}\right] M,
\end{aligned}
$$

where $a_{i}^{[n]}=\sum_{k=0}^{i} a_{k}^{[n-1]}, a_{i}^{[1]}=1, i=0, \ldots, t-1$.

Proof. Let

$$
P=\left[\begin{array}{cc}
A & B \\
0 & 0
\end{array}\right], \quad Q=\left[\begin{array}{cc}
0 & 0 \\
C & D
\end{array}\right]
$$

Then, $M=P+Q$, and, for $n \geq 1$,

$$
\begin{gathered}
Q^{n}=\left[\begin{array}{cc}
0 & 0 \\
D^{n-1} C & D^{n}
\end{array}\right], \quad Q_{d}^{n}=\left[\begin{array}{cc}
0 & 0 \\
D_{d}^{n+1} C & D_{d}^{n}
\end{array}\right], \\
Q^{\pi}=\left[\begin{array}{cc}
I & 0 \\
-D_{d} C & D^{\pi}
\end{array}\right], \quad P^{n}=\left[\begin{array}{cc}
A^{n} & A^{n-1} B \\
0 & 0
\end{array}\right], \\
P_{d}=\left[\begin{array}{cc}
A_{d} & A_{d}^{2} B \\
0 & 0
\end{array}\right], \quad P P_{d}=\left[\begin{array}{cc}
A A_{d} & A_{d} B \\
0 & 0
\end{array}\right]=\left[\begin{array}{cc}
A_{d} & 0 \\
0 & 0
\end{array}\right] M, \\
P^{\pi}=\left[\begin{array}{cc}
A^{\pi} & -A_{d} B \\
0 & I
\end{array}\right], \quad(P+I)_{\alpha}^{n}=\left[\begin{array}{cc}
(A+I)_{\alpha}^{n} & * \\
0 & I
\end{array}\right],
\end{gathered}
$$

where the subscript $\alpha$ stands for $d$ or its absence.

Since $W W_{d}(W+I)=(W+I) W W_{d}, W W_{d}(W+I)_{d}^{n}=$ $(W+I)_{d}^{n} W W_{d}$ where $W$ denotes $P$ or $A$. Thus,

$$
\begin{aligned}
& P P_{d}(P+I)_{\alpha}^{n}=(P+I)_{\alpha}^{n} P P_{d}=\left[\begin{array}{cc}
(A+I)_{\alpha}^{n} A_{d} & 0 \\
0 & 0
\end{array}\right] M, \\
& P P_{d}(P+I)^{\pi}=(P+I)^{\pi} P P_{d}=\left[\begin{array}{cc}
(A+I)^{\pi} & * \\
0 & I
\end{array}\right] P P_{d} \\
& =\left[\begin{array}{cc}
(A+I)^{\pi} A_{d} & 0 \\
0 & 0
\end{array}\right] M, \\
& a_{i}^{[n+2]} I-a_{i}^{[n+1]} Q_{d} \\
& =\left[\begin{array}{cc}
a_{i}^{[n+2]} I & 0 \\
0 & a_{i}^{[n+2]} I
\end{array}\right]-\left[\begin{array}{cc}
0 & 0 \\
a_{i}^{[n+1]} D_{d}^{2} C & a_{i}^{[n+1]} D_{d}
\end{array}\right] \\
& =\left[\begin{array}{cc}
a_{i}^{[n+2]} I & 0 \\
-a_{i}^{[n+1]} D_{d}^{2} C & a_{i}^{[n+2]} I-a_{i}^{[n+1]} D_{d}
\end{array}\right], \\
& P^{i+1} P^{\pi}=\left[\begin{array}{cc}
A^{i+1} & A^{i} B \\
0 & 0
\end{array}\right]\left[\begin{array}{cc}
A^{\pi} & -A_{d} B \\
0 & I
\end{array}\right] \\
& =\left[\begin{array}{cc}
A^{i+1} A^{\pi} & A^{i} A^{\pi} B \\
0 & 0
\end{array}\right] \\
& =\left[\begin{array}{cc}
A^{i} A^{\pi} & 0 \\
0 & 0
\end{array}\right] M, \\
& Q^{n} P^{i+1} P^{\pi}=\left[\begin{array}{cc}
0 & 0 \\
D^{n-1} C & D^{n}
\end{array}\right] P^{i+1} P^{\pi} \\
& =\left[\begin{array}{cc}
0 & 0 \\
D^{n-1} C A^{i} A^{\pi} & 0
\end{array}\right] M .
\end{aligned}
$$


So, for $n \geq 1$,

$$
\begin{gathered}
\left(a_{i}^{[n+2]} I-a_{i}^{[n+1]} Q_{d}\right) Q^{n} P^{i+1} P^{\pi} \\
=\left[\left(\begin{array}{cc}
0 & 0 \\
\left(a_{i}^{[n+2]} I-a_{i}^{[n+1]} D_{d}\right) D^{n-1} C A^{i} A^{\pi} & 0
\end{array}\right] M,\right. \\
\left(a_{i}^{[2]} I-a_{i}^{[1]} Q_{d}\right) P^{i+1} P^{\pi}=\left[\begin{array}{cc}
a_{i}^{[2]} A^{i} A^{\pi} & 0 \\
-a_{i}^{[1]} D_{d}^{2} C A^{i} A^{\pi} & 0
\end{array}\right] M, \\
a_{i}^{[s+1]} Q_{d} Q^{s} P^{\pi} P^{i+1} \\
=a_{i}^{[s+1]}\left[\begin{array}{cc}
0 & 0 \\
D_{d}^{2} C & D_{d}
\end{array}\right]\left[\begin{array}{cc}
0 & 0 \\
D^{s-1} C A^{i} A^{\pi} & 0
\end{array}\right] M \\
0 \\
=\left[\begin{array}{cc}
0 & 0 \\
a_{i}^{[s+1]} D_{d} D^{s-1} C A^{\pi} A^{i} & 0
\end{array}\right] M .
\end{gathered}
$$

Also, for $n \geq 1$,

$$
\begin{aligned}
& Q^{\pi}\left(Q^{n+1}-Q^{n}\right) P P_{d}(P+I)_{d}^{n+2} \\
&=\left[\begin{array}{cc}
I & 0 \\
-D_{d} C & D^{\pi}
\end{array}\right]\left[\begin{array}{cc}
0 & 0 \\
\left(D^{n}-D^{n-1}\right) C & D^{n+1}-D^{n}
\end{array}\right] \\
& \times\left[\begin{array}{cr}
(A+I)_{d}^{n+2} A_{d} & 0 \\
0 & 0
\end{array}\right] M \\
&= {\left[\begin{array}{cc}
0 \\
D^{\pi}\left(D^{n}-D^{n-1}\right) C(A+I)_{d}^{n+2} A_{d} & 0
\end{array}\right] M, }
\end{aligned}
$$

$Q^{\pi}(Q-I) P P_{d}(P+I)_{d}^{2}$

$$
\begin{aligned}
& =\left[\begin{array}{cc}
I & 0 \\
-D_{d} C & D^{\pi}
\end{array}\right]\left[\begin{array}{cc}
-I & 0 \\
C & D-I
\end{array}\right]\left[\begin{array}{cc}
(A+I)_{d}^{2} A_{d} & 0 \\
0 & 0
\end{array}\right] M \\
& =\left[\begin{array}{cc}
-(A+I)_{d}^{2} A_{d} & 0 \\
\left(D_{d}+D^{\pi}\right) C(A+I)_{d}^{2} A_{d} & 0
\end{array}\right] M,
\end{aligned}
$$

$$
\begin{aligned}
\left(Q^{\pi}+\right. & \left.Q_{d}\right) P P_{d}(P+I)_{d} \\
& =\left[\begin{array}{cc}
I & 0 \\
D_{d}^{2} C-D_{d} C & D^{\pi}+D_{d}
\end{array}\right]\left[\begin{array}{cc}
(A+I)_{d} A_{d} & 0 \\
0 & 0
\end{array}\right] M \\
& =\left[\begin{array}{cc}
(A+I)_{d} A_{d} & 0 \\
\left(D_{d}^{2}-D_{d}\right) C(A+I)_{d} A_{d} & 0
\end{array}\right] M,
\end{aligned}
$$

and, for $n \geq 0$,

$$
\begin{aligned}
\left(Q_{d}^{n+1}\right. & \left.-Q_{d}^{n+2}\right) P P_{d}(P+I)^{n}(P+I)^{\pi} \\
= & {\left[\begin{array}{cc}
0 & 0 \\
\left(D_{d}^{n+2}-D_{d}^{n+3}\right) C & D^{n+1}-D^{n+2}
\end{array}\right] } \\
& \times\left[\begin{array}{cc}
(A+I)^{n} A_{d} & 0 \\
0 & 0
\end{array}\right] M\left[\begin{array}{cc}
(A+I)^{\pi} A_{d} & 0 \\
0 & 0
\end{array}\right] M \\
= & {\left[\begin{array}{ccc}
\left(D_{d}^{n+2}-D_{d}^{n+3}\right) C(A+I)^{n}(A+I)^{\pi} A_{d} & 0
\end{array}\right] M . }
\end{aligned}
$$

Since

$$
\begin{gathered}
D-C A_{d} B=D-C B(C B)_{d}^{2} C B D=(C B)^{\pi} D, \\
C A^{\pi}=C-C B C B(C B)_{d}^{2} C=(C B)^{\pi} C,
\end{gathered}
$$

we have

$$
\begin{gathered}
Q_{d} P^{\pi}=\left[\begin{array}{cc}
0 & 0 \\
D_{d}^{2} C & D_{d}
\end{array}\right]\left[\begin{array}{cc}
A^{\pi} & -A_{d} B \\
0 & I
\end{array}\right] \\
=\left[\begin{array}{cc}
0 & 0 \\
D_{d}^{2}(C B)^{\pi} C & D_{d}^{2}(C B)^{\pi} D
\end{array}\right] \\
=\left[\begin{array}{cc}
0 & 0 \\
0 & D_{d}^{2}(C B)^{\pi}
\end{array}\right] M, \\
Q P^{\pi}=\left[\begin{array}{cc}
0 & 0 \\
C A^{\pi} & D-C A_{d} B
\end{array}\right]=\left[\begin{array}{cc}
0 & 0 \\
(C B)^{\pi} C & (C B)^{\pi} D
\end{array}\right], \\
M P^{\pi}=\left[\begin{array}{cc}
A A^{\pi} & B-A A_{d} B \\
C A^{\pi} & D-C A_{d} B
\end{array}\right]=\left[\begin{array}{cc}
A A^{\pi} & B A^{\pi} \\
(C B)^{\pi} C & (C B)^{\pi} D
\end{array}\right] \\
=\left[\begin{array}{cc}
A^{\pi} & 0 \\
0 & (C B)^{\pi}
\end{array}\right] M,
\end{gathered}
$$

and then, by Lemma 4 , $\operatorname{Ind}\left(Q P^{\pi}\right) \leq \operatorname{Ind}\left[(C B)^{\pi} D\right]+1=s$, and $\operatorname{Ind}\left[(P+Q) P^{\pi}\right] \leq \operatorname{Ind}\left[\left[\begin{array}{cc}A^{\pi} & 0 \\ 0 & (C B)^{\pi}\end{array}\right] M\right]=k$.

By Lemma $4, \operatorname{Ind}(P) \leq \operatorname{Ind}(A)+1=t$. Further, by (43), $\operatorname{Ind}\left[(P+I) P P_{d}\right] \leq \operatorname{Ind}\left[(A+I) A A_{d}\right]+1=l$.

Hence, putting (45) (49) into (39) yields (40).

\section{Example}

In this section, we present a numerical example to demonstrate Theorem 7 .

Example 1. Taking $P, Q$ as follows:

$$
\begin{aligned}
P & =\left[\begin{array}{cccc}
0.1321 & 0.8459 & 0.2893 & -0.0597 \\
-0.2830 & 0.0802 & 0.0943 & 0.3066 \\
-0.3396 & 0.1462 & 1.1132 & 0.6179 \\
-0.6226 & 0.9764 & 0.2075 & 0.6745
\end{array}\right], \\
Q & =\left[\begin{array}{cccc}
0.5472 & 1.5283 & 0.1509 & -0.5094 \\
0.0000 & 1.0000 & -0.0000 & 0 \\
0.1132 & -0.3821 & 0.9623 & 0.1274 \\
-0.4528 & 1.5283 & 0.1509 & 0.4906
\end{array}\right],
\end{aligned}
$$

we can get

$$
\begin{gathered}
P_{d}=\left[\begin{array}{cccc}
0.2830 & 0.0031 & 0.2390 & -0.2233 \\
-0.2830 & 0.0802 & 0.0943 & 0.3066 \\
0.8491 & 0.0094 & 0.7170 & -0.6698 \\
-0.8491 & 0.2406 & 0.2830 & 0.9198
\end{array}\right], \\
P^{\pi}=\left[\begin{array}{cccc}
0.9057 & -0.0566 & -0.3019 & 0.0189 \\
0.2830 & 0.9198 & -0.0943 & -0.3066 \\
-0.2830 & -0.1698 & 0.0943 & 0.0566 \\
0.8491 & -0.2406 & -0.2830 & 0.0802
\end{array}\right],
\end{gathered}
$$




$$
\begin{aligned}
Q_{d} & =\left[\begin{array}{cccc}
0.5472 & 1.5283 & 0.1509 & -0.5094 \\
0.0000 & 1.0000 & -0.0000 & 0 \\
0.1132 & -0.3821 & 0.9623 & 0.1274 \\
-0.4528 & 1.5283 & 0.1509 & 0.4906
\end{array}\right], \\
Q^{\pi} & =\left[\begin{array}{cccc}
0.4528 & -1.5283 & -0.1509 & 0.5094 \\
0 & 0 & 0 & 0 \\
-0.1132 & 0.3821 & 0.0377 & -0.1274 \\
0.4528 & -1.5283 & -0.1509 & 0.5094
\end{array}\right],
\end{aligned}
$$

and $\operatorname{Ind}\left(Q P^{\pi}\right)=1, \operatorname{Ind}(P)=2, \operatorname{Ind}\left[(P-I) P P_{d}\right]=2$, and $\operatorname{Ind}\left[(P-Q) P^{\pi}\right]=1$. By Theorem 7 , we have

$$
(P-Q)_{d}=\left[\begin{array}{cccc}
-0.2264 & -0.7358 & 0.0755 & 0.2453 \\
-0.2830 & -0.9198 & 0.0943 & 0.3066 \\
0.1132 & 0.3679 & -0.0377 & -0.1226 \\
-0.1698 & -0.5519 & 0.0566 & 0.1840
\end{array}\right]
$$

\section{References}

[1] N. Castro-González, E. Dopazo, and M. F. Martínez-Serrano, "On the Drazin inverse of the sum of two operators and its application to operator matrices," Journal of Mathematical Analysis and Applications, vol. 350, no. 1, pp. 207-215, 2009.

[2] D. S. Cvetković-Ilić, D. S. Djordjević, and Y. Wei, "Additive results for the generalized Drazin inverse in a Banach algebra," Linear Algebra and Its Applications, vol. 418, no. 1, pp. 53-61, 2006.

[3] R. E. Hartwig, G. Wang, and Y. Wei, "Some additive results on Drazin inverse," Linear Algebra and Its Applications, vol. 322, no. 1-3, pp. 207-217, 2001.

[4] X. Liu, L. Xu, and Y. Yu, “The representations of the Drazin inverse of differences of two matrices," Applied Mathematics and Computation, vol. 216, no. 12, pp. 3652-3661, 2010.

[5] A. S. Cvetković and G. V. Milovanović, "On Drazin inverse of operator matrices," Journal of Mathematical Analysis and Applications, vol. 375, no. 1, pp. 331-335, 2011.

[6] G. Wang, Y. Wei, and S. Qiao, Generalized Inverses: Theory and Computations, Science Press, Beijing, China, 2004.

[7] C. Y. Deng, "The Drazin inverse of bounded operators with commutativity up to a factor," Applied Mathematics and Computation, vol. 206, no. 2, pp. 695-703, 2008.

[8] C. Y. Deng, "The Drazin inverses of sum and difference of idempotents," Linear Algebra and Its Applications, vol. 430, no. 4, pp. 1282-1291, 2009.

[9] S. L. Campbell and C. D. Meyer Jr., Generalized Inverse of Linear Transformations, Pitman, Boston, Mass, USA, 1991.

[10] M. P. Drazin, "Pseudo-inverses in associative rings and semigroups," The American Mathematical Monthly, vol. 65, pp. 506$514,1958$.

[11] C. D. Meyer Jr. and N. J. Rose, "The index and the Drazin inverse of block triangular matrices," SIAM Journal on Applied Mathematics, vol. 33, no. 1, pp. 1-7, 1977. 


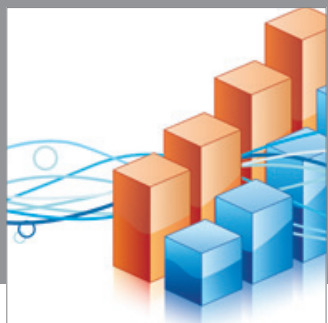

Advances in

Operations Research

mansans

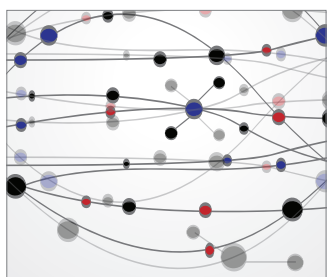

The Scientific World Journal
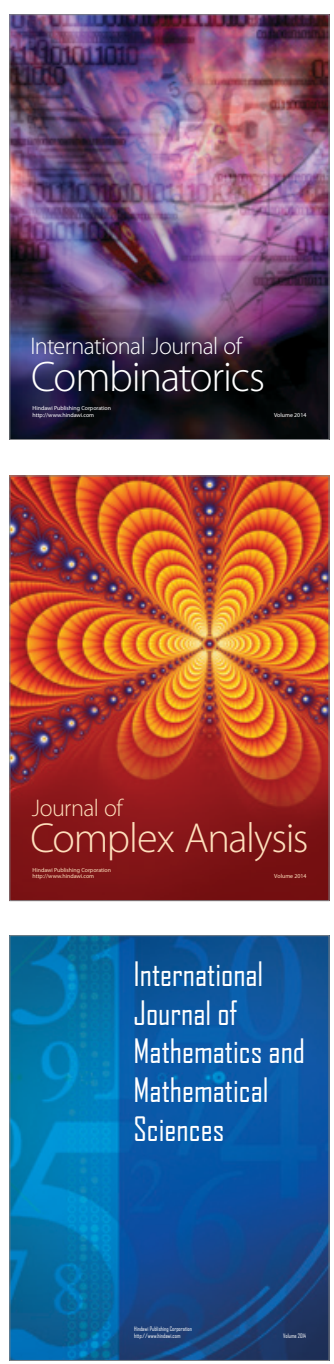
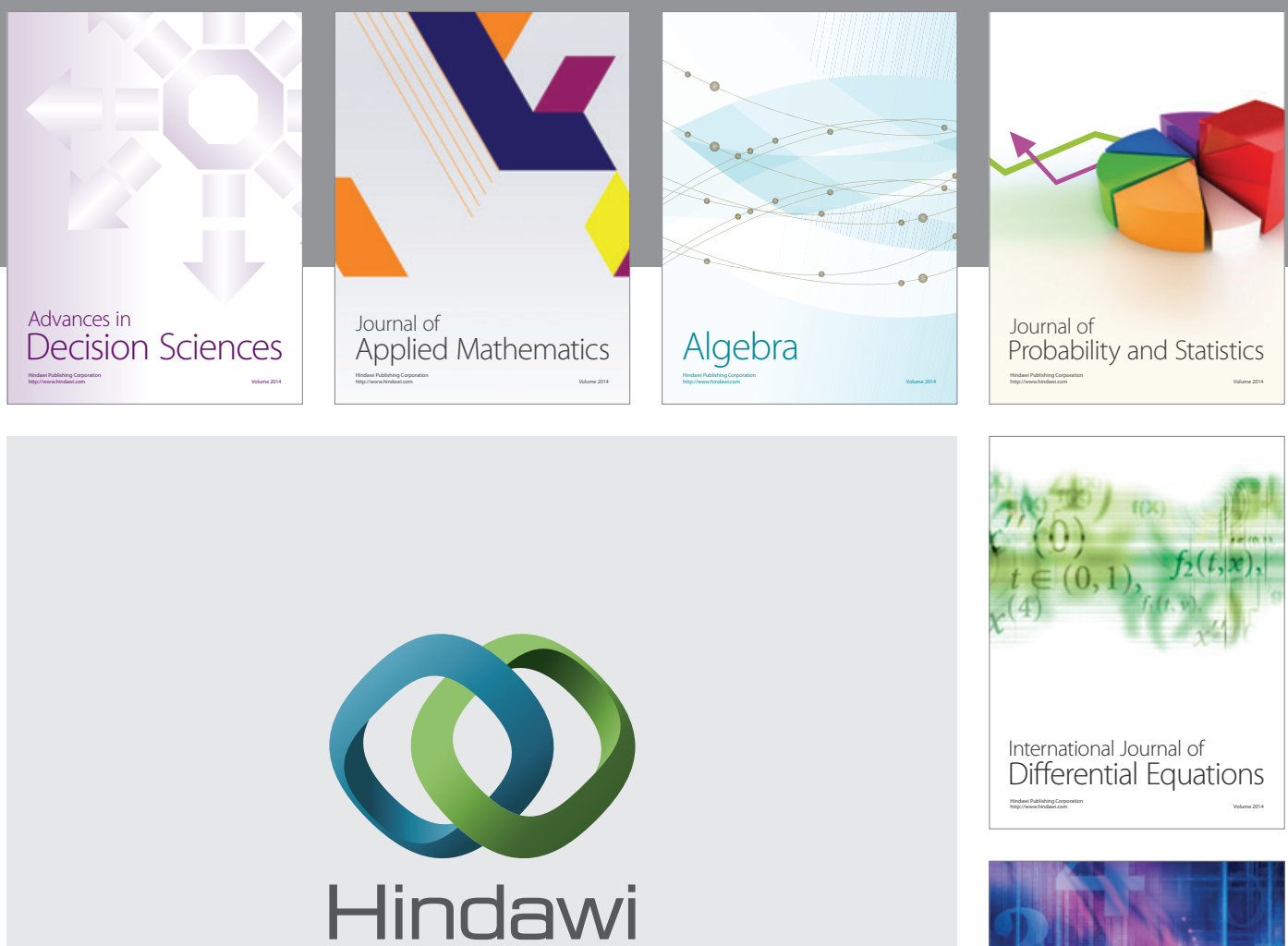

Submit your manuscripts at http://www.hindawi.com
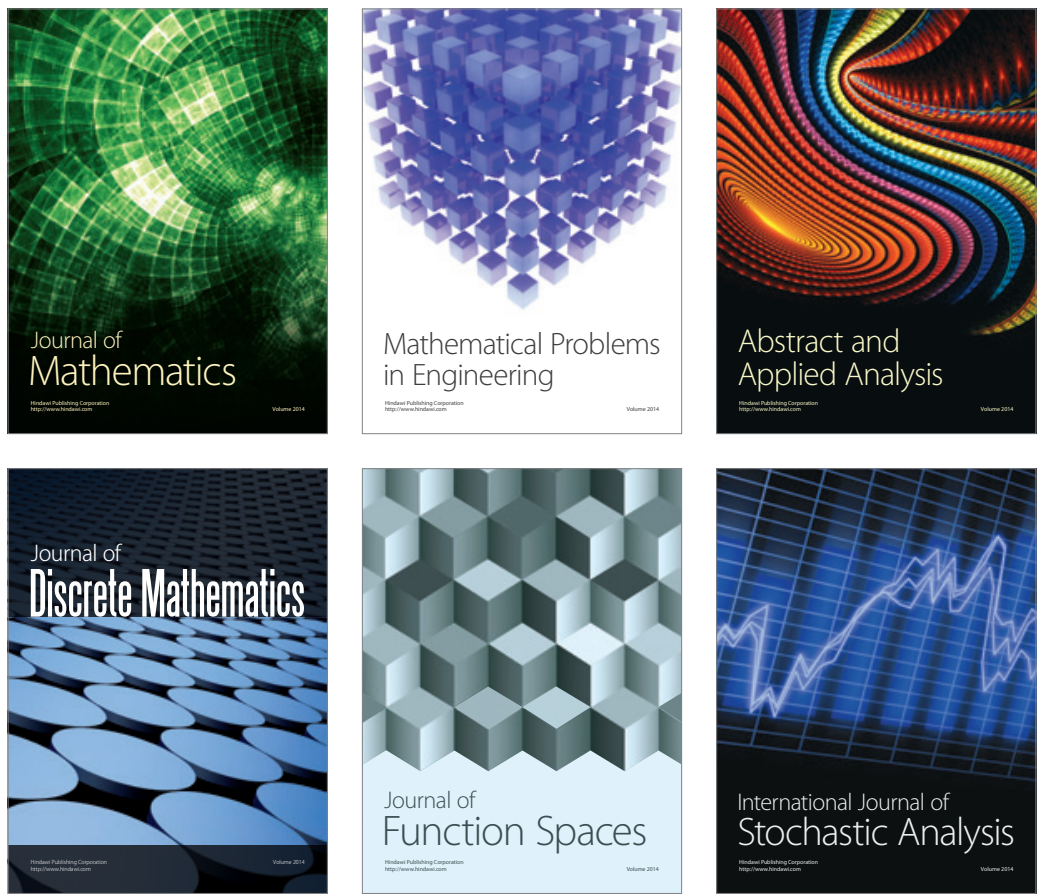

Journal of

Function Spaces

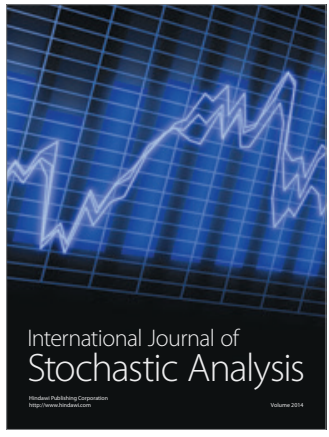

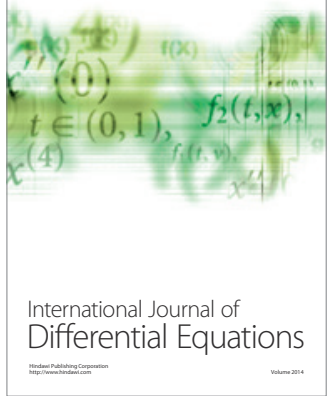
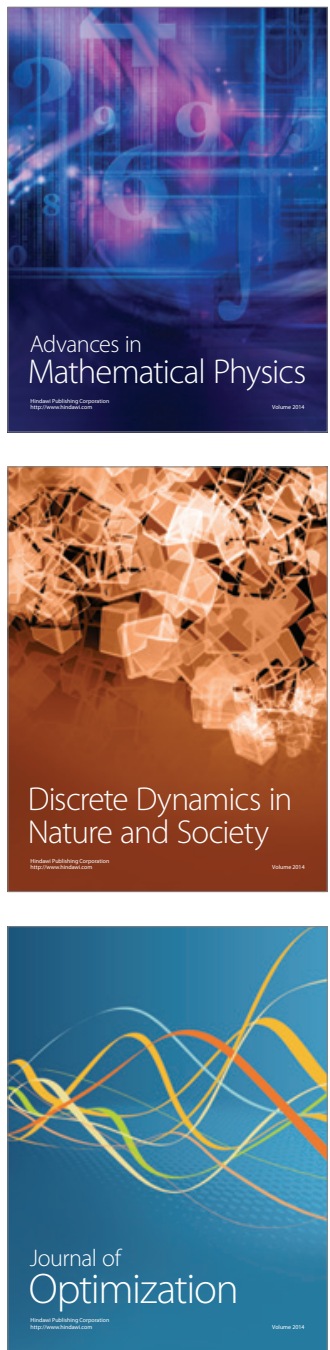